\title{
A comparative study on the processing, dielectric and electrical properties of PMN based solid solutions
}

\author{
KAMAL SINGH* and SHEELA BAND ${ }^{\dagger}$ \\ Department of Physics, Amravati University, Amravati 444602 , India \\ ${ }^{\dagger}$ Department of Applied Physics, Y. C. Engineering College, Wanadongari 441 110, India \\ MS received 9 April 1996; revised 13 November 1996
}

\begin{abstract}
Lead magnesium niobate (PMN) based binary with lead titanate (PT), lead iron niobate (PFN) and lead zinc niobate (PZN) and ternary with PZN-PT and PFN-PT solid solutions have been synthesized. In depth characterization using $\mathrm{X}$-ray diffraction and SEM techniques have revealed the major perovskite phase formation. Amongst all the solid solutions, PMN-PZN-PT has given the highest values of permittivity as 19,740 and 23,700 with $T_{\mathrm{c}}$ as $34^{\circ} \mathrm{C}$ when sintered at $1080^{\circ} \mathrm{C}$ and $1180^{\circ} \mathrm{C}$ respectively on the one hand and on the other, PFN containing solid solutions exhibited distinct deviation from the relaxor behaviour.
\end{abstract}

Keywords. Solid solution; sintering; relaxor; microstructure.

\section{Introduction}

In the late 1950s Smolenskii and Agranovskaya (1959), discovered a family of lead based perovskites with general formula $\mathrm{A}\left(\mathrm{B}^{\prime} \mathrm{B}^{\prime \prime}\right) \mathrm{O}_{3}$ exhibiting diffuse phase transition (DPT). Many of these DPT materials are relaxors offering very high relative permittivity in the range $8000-25,000$ which have been of a great commercial importance due to their applications in multilayer capacitors, electrostatic actuators, pyroelectric detectors, bolometers etc.

Amongst the lead based relaxors, PMN and PZN have exhibited high relative permittivity (14,000-20,000) (Swartz et al 1984; Lejeune and Boilot 1985; Wang and Schulze 1990; Singh and Band 1996) and 22,000 (Bokov and Mylnikova 1961) respectively. But PMN has been most widely studied as compared to PZN owing to relative ease of preparation as the latter was found to be extremely difficult to obtain in the pure perovskite ceramic form by conventional mixed oxide method or as suggested by Swartz and Shrout (1982). The ferroelectric transition $T_{\mathrm{c}}$ of PMN below room temperature $\left(-8\right.$ to $\left.-15^{\circ} \mathrm{C}\right)$ has been one of the factors which constraints its practical utilization.

Several authors have shown that the substitutional effect of PT in PMN resulted in the rise in permittivity, shift of $T_{\mathrm{c}}$ towards room temperature and also an improvement in the extent of perovskite phase formation (Swartz et al 1984; Yan et al 1989; Bouquin and Lejeune 1991; Ravindranathan et al 1991). Landin and Schulze (1990) observed rapid densification in case of $0.7 \mathrm{PMN}-0.3 \mathrm{PZN}$ solid solution at $1100^{\circ} \mathrm{C}$. Furthermore, in this system, the maximum permittivity at $20^{\circ} \mathrm{C}$ has also been obtained. Desgardin et al (1983), in their investigation on ceramic compositions based on PMN have obtained $T_{c}$ around $20^{\circ} \mathrm{C}$ for a particular composition 0.77 PMN-0.23 PFN. In addition to this, the use of selective flux therein offered them weak dielectric loss and high resistivity.

\footnotetext{
*Author for correspondence
} 
Table 1. Composition and designation of PMN based solid solutions.

\begin{tabular}{llcc}
\hline Composition & Constituents & $\begin{array}{c}\text { Sintering } \\
\text { temperature } \\
\left({ }^{\circ} \mathrm{C}\right)\end{array}$ & Designation \\
\hline 0-9PMN-0.1PT & PbO, MN, TiO ${ }_{2}$ & 1080 & PMT1 \\
0-7PMN-0-3PZN & & 1180 & PMT2 \\
& PbO, MN, ZN & 1080 & PMZN1 \\
0-7PMN-0-3PFN & & 1180 & PMZN2 \\
& & 1080 & PMFN1 \\
0.85PMN-0.07PZN-0.08PT & PbO, MN, ZN, TiO 2 & 1180 & PMFN2 \\
& & 1080 & PMZNT1 \\
0-85PMN-0.07PFN-0-08PT & PbO, MN, FN, TiO 2 & 1180 & PMZNT2 \\
& & 1080 & PMFNT1 \\
\hline
\end{tabular}

Eventhough there have been several reports on the investigations of PMN based solid solutions but still a comparative study on the sintering temperature, grain size, relative permittivity, low losses, electrical resistivity and $T_{\mathfrak{c}}$ nearing room temperature in the summarized form is of importance. Hence, in the present study we have made an attempt to undertake a comparative study on the processing of PMN based binary and ternary solid solutions such as PMN-PT, PMN-PZN, PMN-PFN, PMN-PZNPT and PMN-PFN-PT following a structural characterization, dielectric and electrical properties with a focus on the microstructures.

\section{Experimental}

Ceramics of the selected compositions shown in table 1 have been synthesized using Swartz's technique (Swartz and Shrout 1982). The initial ingradients $\mathrm{PbO}, \mathrm{MgO}, \mathrm{ZnO}$, $\mathrm{Nb}_{2} \mathrm{O}_{5}, \mathrm{Fe}_{2} \mathrm{O}_{3}$ and $\mathrm{TiO}_{2}$ used have been of $99.9 \%$. Initially, the coulumbites $\mathrm{MgNb}_{2} \mathrm{O}_{6}$ and $\mathrm{ZnNb}_{2} \mathrm{O}_{6}$ have been prepared by taking $\mathrm{MgO}: \mathrm{Nb}_{2} \mathrm{O}_{5}$ in $1.02: 1$ and $\mathrm{ZnO}: \mathrm{Nb}_{2} \mathrm{O}_{5}$ in $1: 1$ molar ratios, followed by calcination and re-calcination at $1000^{\circ} \mathrm{C}$ for 4 and $2 \mathrm{~h}$ respectively. Later wolframite $\mathrm{FeNbO}_{4}$ has been prepared by taking $\mathrm{Fe}_{2} \mathrm{O}_{3}$ and $\mathrm{Nb}_{2} \mathrm{O}_{5}$ in $1: 1$ molar ratio and calcining for $4 \mathrm{~h}$ at $1000^{\circ} \mathrm{C}$. Thus prepared $\mathrm{MgNb}_{2} \mathrm{O}_{6}, \mathrm{ZnNb}_{2} \mathrm{O}_{6}$ and $\mathrm{FeNbO}_{4}$ have been designated as $\mathrm{MN}, \mathrm{ZN}$ and $\mathrm{FN}$ respectively. Finally the solid solutions of above mentioned compositions have been prepared by taking presynthesized constituent oxides in appropriate molar ratios followed by a number of processing steps as shown in figure 1. In order to overcome $\mathrm{PbO}$ losses, the pellets have been surrounded by the powder of the same composition. Various ceramics prepared have been designated as given in table 1. The bulk densities of sintered pellets have been measured using Archimedes principle.

X-ray diffractometer (XRD) scans have been recorded on powder of sintered pellets with the help of PW1700 X-ray powder diffractometer using iron target $(\lambda=1.93735 \AA)$ at the scanning rate of $2 \theta=2.4^{\circ} / \mathrm{min}$ at RSIC, Nagpur. The fractured surface of the 


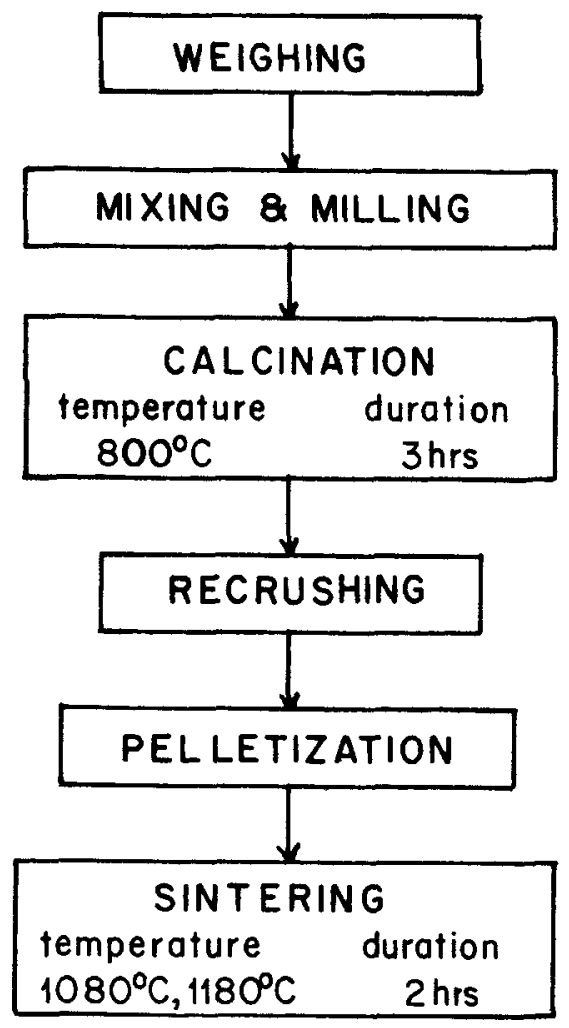

Figure 1. Flowchart of material processing of PMN based solid solutions.

sintered pellets has been examined by using Cambridge 250 Mark (III) scanning electron microscope (SEM) at RSIC, Nagpur.

The relative permittivity $(\varepsilon)$ and dissipation factor $(\tan \delta)$ have been measured using HP 4192A LF impedance analyser, pseudo-continuously in the frequency range from 0.1 to $100 \mathrm{kHz}$ and temperature ranging from -50 to $100^{\circ} \mathrm{C}$. The dc resistivity at room temperature has been measured using Keithley 617 programmable electrometer with an electric field $150 \mathrm{~V} / \mathrm{cm}$ being applied across the samples.

\section{Results and discussion}

\subsection{XRD and SEM characterization}

The XRD patterns of PMT2, PMZN2, PMZNT2, PMFN2 and PMFNT2 are shown in figure 2. An excellent agreement between experimental 'd' values with standard JCPDS (File 27-1199) ones corresponding to PMN confirms perovskite phase formation. A critical analysis of XRD data reveals cubic symmetry of the perovskite phase in all samples. The relative \% of perovskite phase formation is estimated using (1) below (Swartz and Shrout 1982; Wang and Schulze 1990; Singh and Band 1996),

$$
\% \text { perovskite }=\frac{\text { Iperov }(110)}{\text { Iperov (110) }+ \text { Ipyro (222) }}
$$




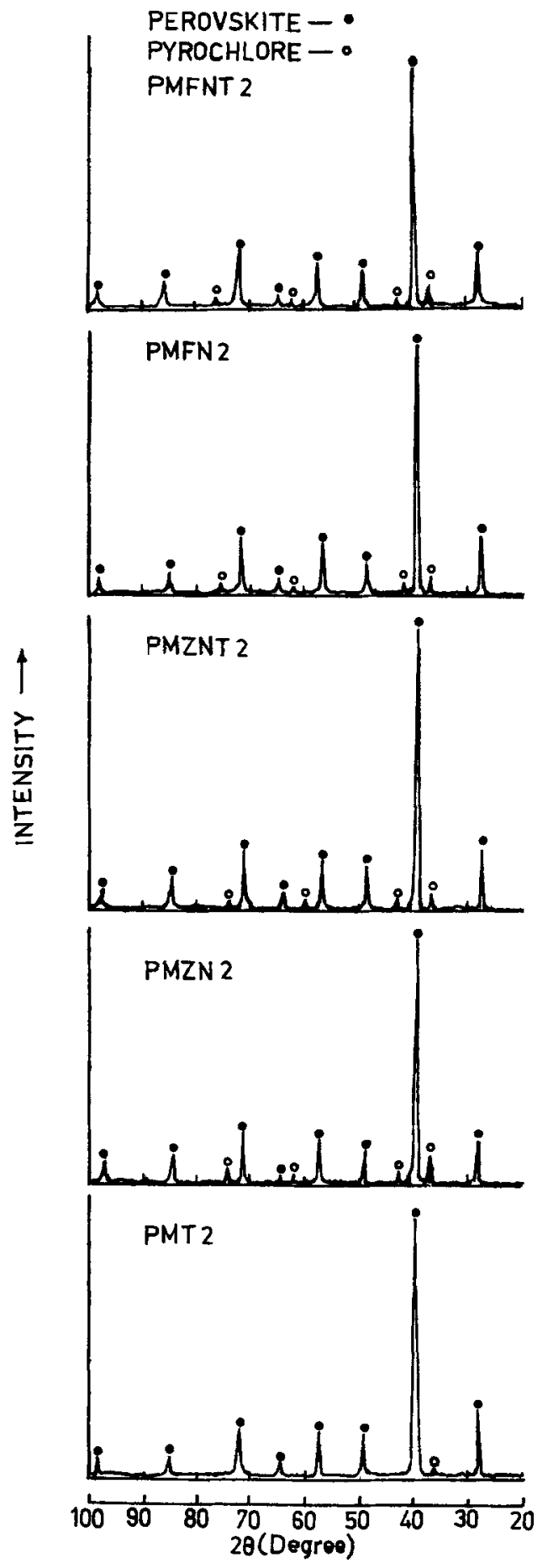

Figure 2. X-ray powder diffraction patterns of PMT2, PMZN2, PMZNT2, PMFN2 and PMFNT2. 
Table 2. Perovskite phase (\%) and lattice cell constant $a$ of $\mathrm{PMN}$ based solid solutions.

\begin{tabular}{lcc}
\hline Sample & $\begin{array}{c}\text { Perovskite } \\
(\%)\end{array}$ & $\begin{array}{c}\text { Lattice constant } \\
(\AA)\end{array}$ \\
\hline PMT1 & 96.5 & $4.041 \pm 0.001$ \\
PMT2 & 98.5 & $4.041 \pm 0.001$ \\
PMZN2 & 91.0 & $4.056 \pm 0.001$ \\
PMFN1 & 92.0 & $4.042 \pm 0.001$ \\
PMFN2 & 94.5 & $4.042 \pm 0.001$ \\
PMZNT2 & 95.0 & $4.040 \pm 0.001$ \\
PMFNT1 & 94.0 & $4.042 \pm 0.001$ \\
PMFNT2 & 93.0 & $4.041 \pm 0.001$ \\
\hline
\end{tabular}

where, Iperov and Ipyro are the intensities of (110) and (222) characteristic lines respectively.

The $\%$ of perovskite phase along with the lattice cell constant values determined from high angle reflections are given in table 2 . In addition to the absence of characteristics lines corresponding to solute phase, the variation of lattice cell constant $a$ from $4.039 \pm 0.001$ to $4.056 \pm 0.001$ ensures the solid solution formation in all compositions. A systematic correlation of lattice cell constant with composition can not be predicted precisely. However, a comparatively high lattice cell constant obtained for PMZN2, which contains high concentration of $\mathrm{Zn}$ ( 30 mole \%), is due to larger radius of $\mathrm{Zn}(0.74 \AA)$ replacing smaller $\mathrm{Mg}(0.65 \AA)$ in the B-site of $\mathrm{ABO}_{3}$ perovskite structure. Goldschmidt (1926) concluded that, for the perovskite structure formation, tolerance factor should be within 0.88 to 1.09 . The large value of tolerance factor indicates higher stability of perovskite phase. The values of tolerance factor for the perovskite compounds reported by Shrout and Halliyal (1987), follow the PZN $<$ PMN $<$ PFN $<$ PT sequence with respective values of $0.984,0.99,1.0,1.02$. In the present case, the improvement in the perovskite phase is observed as a result of increased tolerance factor of the corresponding binary solid solution (table 2). Interestingly, it is observed that the addition of PT to PMN as well as to PMN-PZN solid solution stabilizes the perovskite phase (table 2) which is in good agreement with earlier findings (Bouquin and Lejeune 1991; Ravindranathan et al 1991).

The microphotographs of PMT2, PMZN1, PMZN2, PMFN2, PMZNT2 and PMFNT2 are displayed in figures $3 \mathrm{a}-\mathrm{f}$ respectively. The grain size calculated from microphotographs using ASTM intercept technique is presented in table 3. A close look at these microphotographs reveals almost a similar morphology of the grains of all the samples confirming the maximum perovskite phase formation supporting strongly the XRD results.

In some samples a few distinct grains of pyrochlore, the second phase, are clearly seen. Furthermore, as a general behaviour of lead based perovskites, in all compositions the increased grain size with increasing sintering temperature is observed. A comparatively large perovskite grains are observed for PMN-PZN and PMNPFN binary solid solutions. The PMN-PT solid solution, whereas, exhibits relatively smaller grains. The decrease in grain size with PT addition is observed in both PMN-PZN and PMN-PFN binary solid solutions (table 3). 


\subsection{Dielectric properties}

The $\varepsilon_{\max }, T_{\mathrm{c}}, \tan \delta_{\max }$ and $T \tan \delta_{\max }$ at $0 \cdot 1,1,10$ and $100 \mathrm{kHz}$ of all the samples are presented in table 4. The temperature dependence of $\varepsilon$ and $\tan \delta$ at $0 \cdot 1,1,10$ and $100 \mathrm{kHz}$ of typical samples PMZNT2 and PMFN2 are shown in figures 4 and 5 respectively.
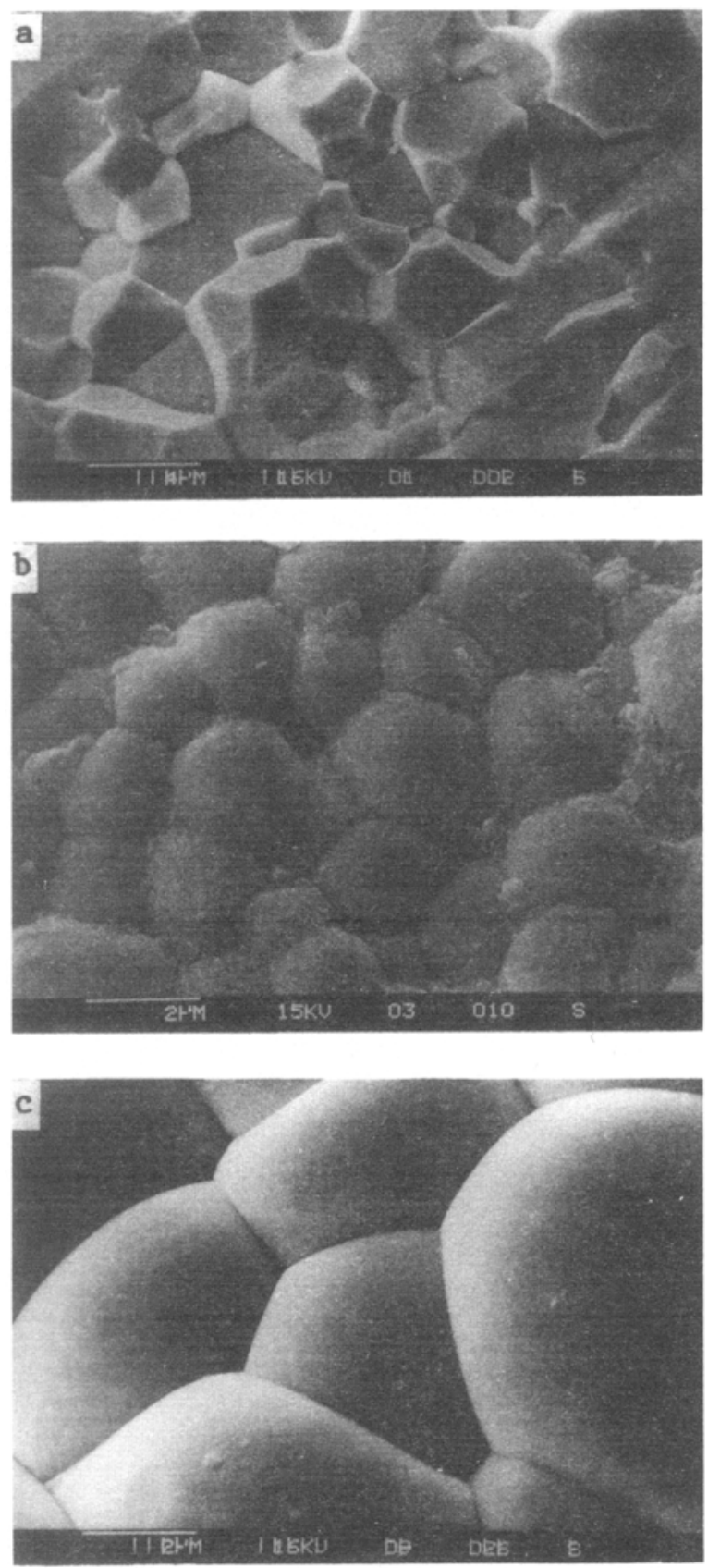

Figure 3. a-c. 

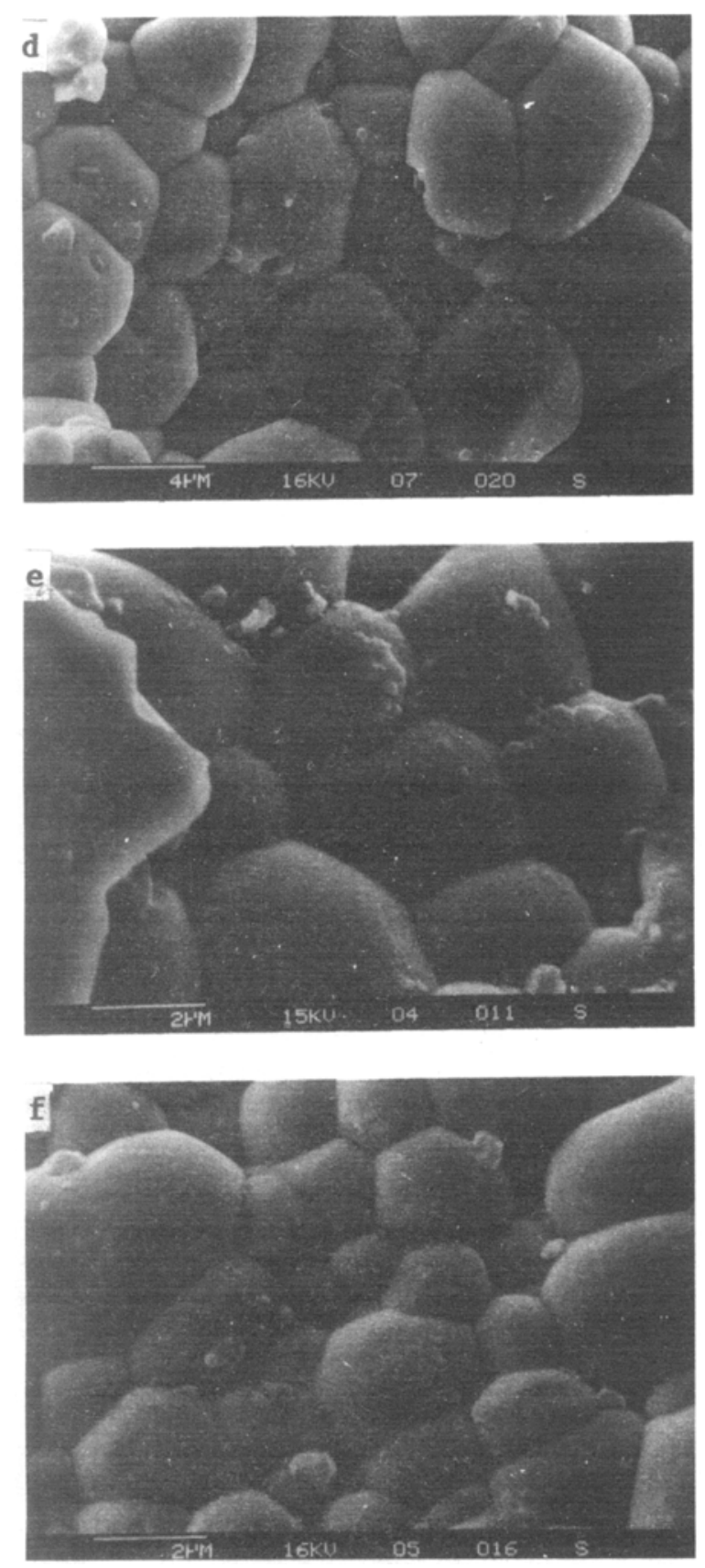

Figure 3. SEM photographs of fractured surface of (a) PMT2, (b) PMZN1, (c) PMZN2, (d) PMFN2, (e) PMZNT2 and (f) PMFNT2.

The samples PMT1, PMT2, PMZN1, PMZN2, PMZNT1 and PMZNT2 clearly exhibit a typical relaxor behaviour (figure 4). Increase in frequency decreases the magnitude of $\varepsilon_{\max }$ on one hand and increases $\tan \delta_{\max }$ on the other and shifts the maxima in $\varepsilon$ and $\tan \delta$ towards higher temperature. The $\tan \delta_{\max }$ appears at a 
Table 3. Grain size of perovskite phase and densification of PMN based solid solutions.

\begin{tabular}{lcc}
\hline Sample & $\begin{array}{c}\text { Grain size } \\
(\mu \mathrm{m})\end{array}$ & $\begin{array}{c}\text { Densification } \\
(\%)\end{array}$ \\
\hline PMT1 & $2 \cdot 1 \pm 0 \cdot 2$ & 94 \\
PMT2 & $4 \cdot 9 \pm 0 \cdot 2$ & 98 \\
PMZN1 & $2 \cdot 9 \pm 0 \cdot 2$ & 95 \\
PMZN2 & $6 \cdot 9 \pm 0 \cdot 6$ & 96 \\
PMFN1 & $2 \cdot 2 \pm 0 \cdot 2$ & 93 \\
PMFN2 & $6 \cdot 6 \pm 0 \cdot 3$ & 97 \\
PMZNT1 & - & 95 \\
PMZNT2 & $4 \cdot 4 \pm 0 \cdot 3$ & 97 \\
PMFNT1 & $1 \cdot 2 \pm 0 \cdot 3$ & 93 \\
PMFNT2 & $3 \cdot 5 \pm 0 \cdot 4$ & 97 \\
\hline
\end{tabular}

temperature range lower than that corresponding to $\varepsilon_{\max }$, indicating low losses near ambient temperature. The samples PMFN1, PMFN2, PMFNT1 and PMFNT2 show a systematic frequency dispersion in $\varepsilon_{\max }$ with temperature except the missing transition at $100 \mathrm{~Hz}$ in case of PMFN2 (figure 5). In contrast, no systematic variation of $\tan \delta$ with temperature and frequency is observed, reflecting a deviation from the relaxor behaviour. The deviation from relaxor behaviour is found to be more pronounced for higher concentration of $\mathrm{Fe}$ and sintering temperature (figure 5). Low losses comparable to PMN are observed in case of PMT1, PMT2, PMZN1, PMZN2, PMZNT1, PMZNT2 and PMFNT1 samples. Whereas, slightly higher losses in PMFNT2 and substantially high losses are detected in PMFN1 and PMFN2 samples.

It is well known that in relaxors, there exists inbuilt compositional microinhomogeneity amongst individual unit cells. Such a microinhomogeneity results into a small variation in the potential energy of ferroactive B-site atoms of oxygen octahedra responsible for temperature sensitive atomic displacement and variation in relaxation time. A statistical average of identical compositional unit cells which have a transition temperature with a fixed relaxation time. Likewise, there could be different groups corresponding to similar compositions having same relaxation time. Therefore, with the change in frequency, $T_{c}$ changes as each will have different relaxation frequency as reflected in figure 4 . Anologously inbuilt heterogeneity in compositions within unit cell to unit cell results into variation in soft modes that spreads over the temperature region giving rise to DPT behaviour.

For all compositions it is seen that an increase in sintering temperature (table 4) increases the permittivity maximum but lowers the transition temperature. A closer look at table 4 reveals that PMZNT1 (sintered at $1080^{\circ} \mathrm{C}$ ) and PMZNT2 (sintered at $1180^{\circ} \mathrm{C}$ ) exhibit highest permittivity maximum equal to 19740 and 23700 respectively. It is worth noting here that PMZNT1 $\left(T_{c}=34.5^{\circ} \mathrm{C}\right)$ gave almost same value of permittivity maximum as that of $\mathrm{PMN}\left(T_{\mathrm{c}}=-16^{\circ} \mathrm{C}\right)$ sintered at $1200^{\circ} \mathrm{C}$ (Wang and Schulze 1990). Interestingly, the permittivity maximum obtained in PMT2 is found to be comparable with that of reported permittivity maximum $=20372$ for gel derived ceramics (Ravindranathan et al 1991). Also, the permittivity maximum obtained in PMZN1 is observed to be significantly higher than the reported value of the order 14400 (Landin and Schulze 1990). On the other hand 0-9PMN-0.1PT and 0.85PMN-0.07PFN-0.08PT exhibit reasonably high permittivity maximum provided they are sintered at higher temperature $\left(\geqslant 1150^{\circ} \mathrm{C}\right)$ (table 4$)$. 


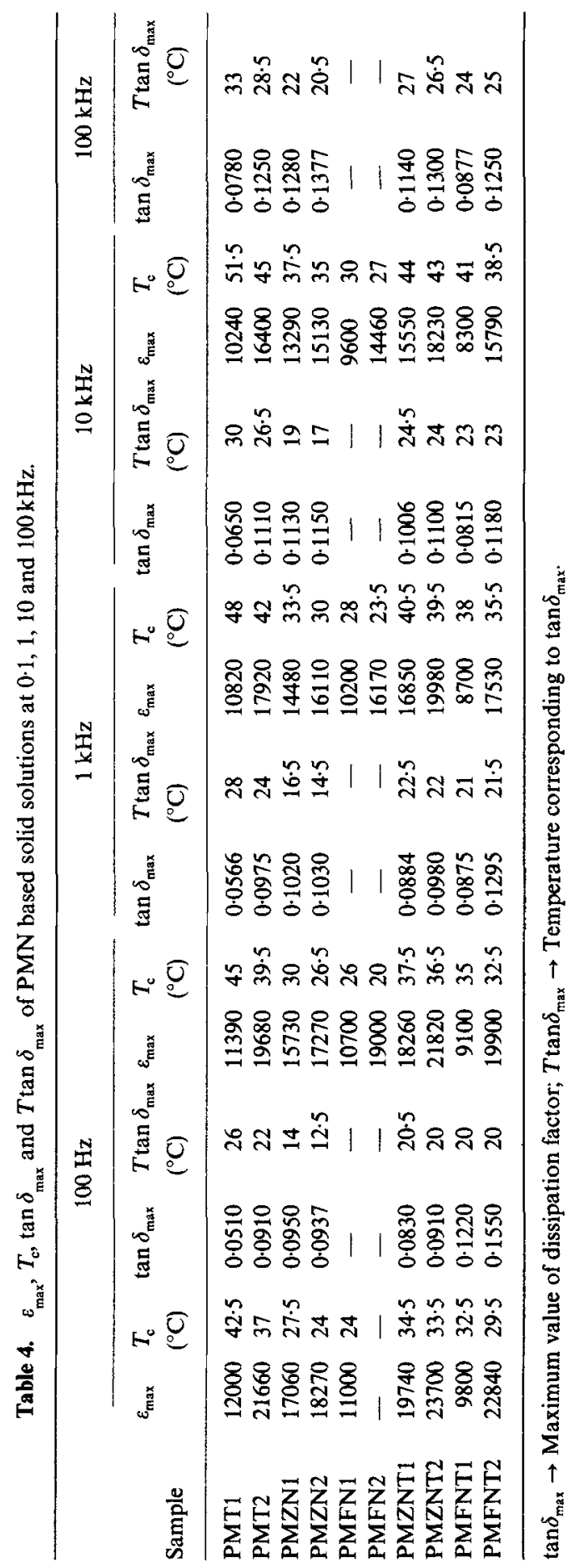




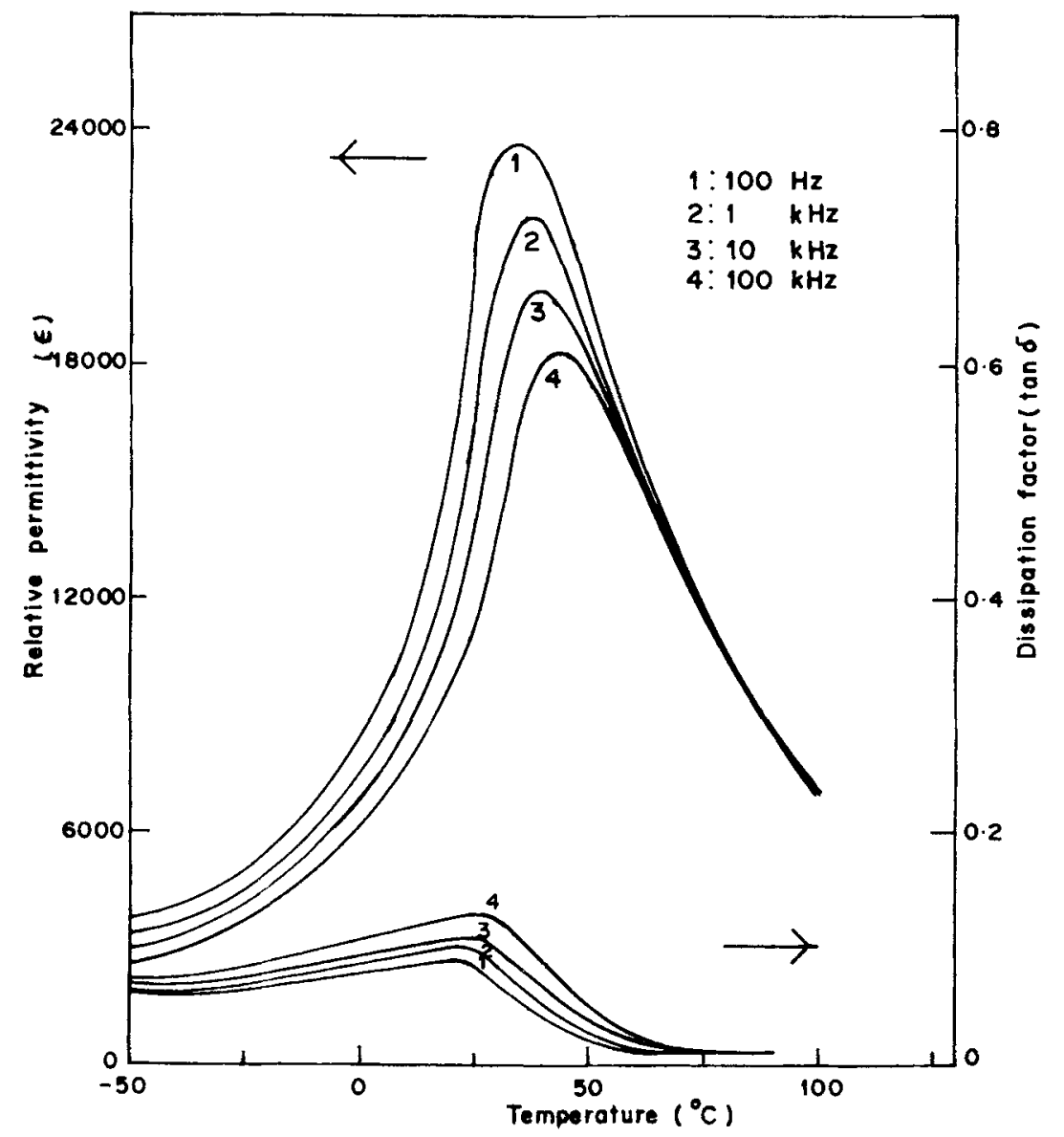

Figure 4. Temperature dependence of relative permittivity $(\varepsilon)$ and dissipation factor $(\tan \delta)$ of PMZNT2 at $0 \cdot 1,1,10$ and $100 \mathrm{kHz}$.

As stated earlier our interest was in developing the relaxors with high permittivity and $T_{\mathrm{c}}$ near room temperature. Hence the compositions have been selected for the present study wherein, $T_{\mathrm{c}}$ ranged within 24 to $42 \cdot 5^{\circ} \mathrm{C}$ at $\mathrm{Hz}$ (table 4 ).

The highest permittivity maximum obtained for PMZNT2 is apparently due to improved perovskite phase, higher density and high intrinsic permittivity of PZN. In PMFN2, the anamoly in high permittivity seems to be a result of space charge polarization arising due to oxygen vacancies during sintering process leading to partial conversion of $\mathrm{Fe}^{3+}$ to $\mathrm{Fe}^{2+}$ in the interfaces of the grain boundaries. $\mathrm{The}^{\mathrm{Fe}} \mathrm{e}^{3+}$ to $\mathrm{Fe}^{2+}$ conversion is supported with the naked eye observation of the change in colour from brown to black seen in cases of PMFN1 and PMFN2. The above results are in total agreement with the suggested model (Ichinose and Kato 1994; Yokosuka 1995). Hence in conclusion, the increase in $\mathrm{Fe}$ concentration and sintering temperature together promote the conversion of $\mathrm{Fe}^{3+}$ to $\mathrm{Fe}^{2+}$ leading to increase in $\varepsilon$ and high losses.

The quadratic law,

$$
\frac{1}{\varepsilon}=\frac{1}{\varepsilon_{\max }}+\left(T-T_{\mathrm{c}}\right)^{2} / 2 \varepsilon_{\max } \delta^{2},
$$




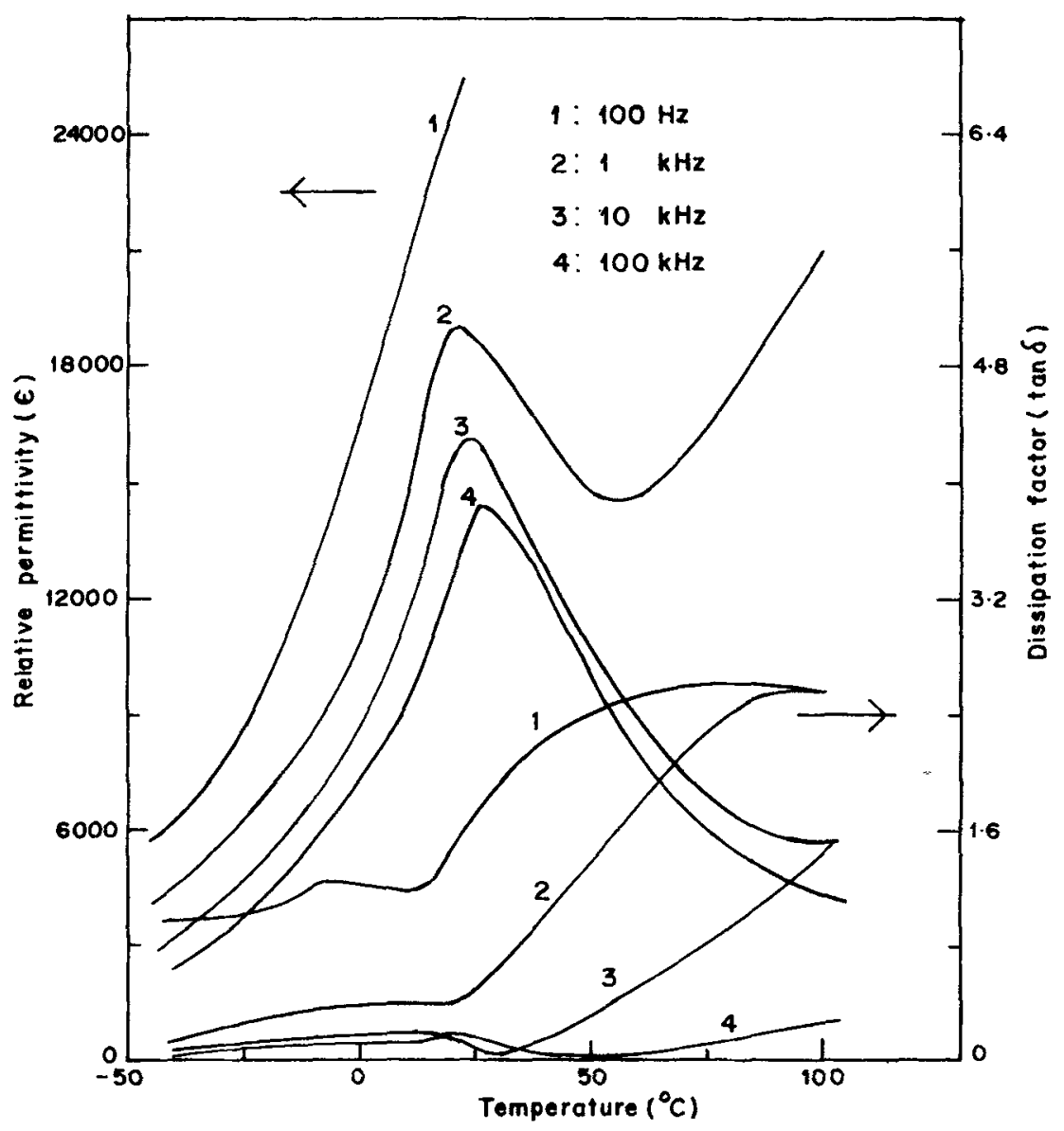

Figure 5. Temperature dependence of relative permittivity $(\varepsilon)$ and dissipation factor $(\tan \delta)$ of PMFN2 at $0 \cdot 1,1,10$ and $100 \mathrm{kHz}$.

where, $T$ and $\delta$ are temperature above $T_{\mathrm{c}}$ and diffusion coefficient respectively established for DPT materials is found to be valid over a temperature range of investigation for all samples except PMFN1 and PMFN2.

The plots of $1 / \varepsilon$ vs $\left(T-T_{\mathrm{c}}\right)^{2}$ for PMFN1, PMFN2 and PMZNT1, PMZNT2 samples are depicted in figures $6 \mathrm{a}-\mathrm{d}$ respectively. The large deviation from linearity is observed with increased concentration of $\mathrm{Fe}$ at higher sintering temperature (figure $6 \mathrm{~b}$ ). The diffusion coefficient calculated using (2) at $1 \mathrm{kHz}$ along with the difference between transition temperature $\Delta T_{\mathrm{c}}$ measured at 0.1 to $100 \mathrm{kHz}$ i.e. curie range, are presented in table 5 . The general trend of decrease in diffusion coefficient with increase in permittivity maximum is observed in case of 0.9PMN-0.1PT and 0.85PMN-0.07PFN-0-08PT solid solutions. However 0.7PMN-0.3PZN shows opposite behaviour, whereas, no detectable change is observed in case of $0.85 \mathrm{PMN}-0.07 \mathrm{PZN}-0.08 \mathrm{PT}$ solid solution.

\subsection{Sintered density and dc resistivity}

The values of sintered density of all the samples are presented in table 3. As expected, higher density is achieved in all compositions of solid solution sintered at higher 


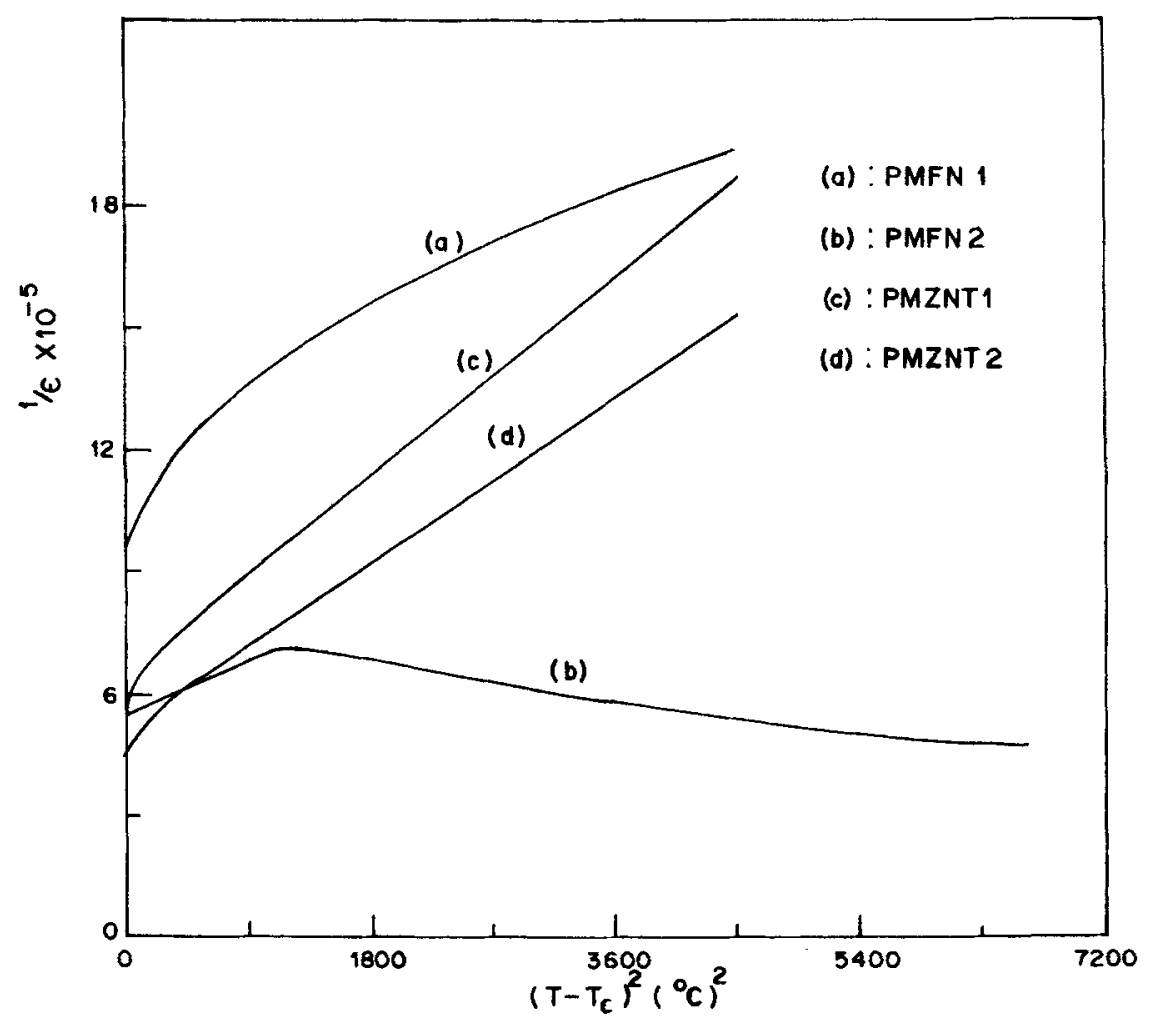

Figure 6. Plots of reciprocal of relative permittivity $1 / \varepsilon$ vs $\left(T-T_{\mathrm{c}}\right)^{2}$ at $1 \mathrm{kHz}$ for (a) PMFN1, (b) PMFN2, (c) PMZNT1 and (d) PMZNT2.

Table 5. Diffusion coefficient $(\delta)$ and curie range $\left(\Delta T_{\mathrm{c}}\right)$ of $\mathrm{PMN}$ based solid solutions.

\begin{tabular}{lcc}
\hline Sample & $\begin{array}{c}\delta \\
\left({ }^{\circ} \mathrm{C}\right)\end{array}$ & $\begin{array}{c}\Delta T_{\mathrm{c}} \\
\left({ }^{\circ} \mathrm{C}\right)\end{array}$ \\
\hline PMT1 & 45 & 9 \\
PMT2 & 37 & 8 \\
PMZN1 & 35 & 10 \\
PMZN2 & 41 & 11 \\
PMZNT1 & 32 & $9 \cdot 5$ \\
PMZNT2 & 32 & $9 \cdot 5$ \\
PMFNT1 & 55 & 9 \\
PMFNT2 & 33 & $8 \cdot 5$ \\
\hline
\end{tabular}

temperature. It is worth noting that $95 \%$ of theoretical density is obtained for PMZN1 and PMZNT1 samples inspite of sintering at low temperature $\left(1080^{\circ} \mathrm{C}\right)$. Furthermore, $96 \%$ density obtained for $\mathrm{PMZN} 2$ sintered at $1180^{\circ} \mathrm{C}$ indicates attainment of saturation in density in case of $0.7 \mathrm{PMN}-0.3 \mathrm{PZN}$ solid solution. These results are similar to those of Landin and Schulze (1990). A relatively lower density ( $96 \%$ ) obtained for PMZN2 is a consequence of higher content of low density pyrochlore phase present therein. 
In general, the d.c. resistivity at room temperature is found to be $\geqslant 10^{12} \mathrm{ohm}-\mathrm{cm}$ except for PMFN1, PMFN2 and PMFNT2. However, PMFN1, PMFN2 and PMFNT2 shows resistivity of the order of $10^{7}, 10^{5}$ and $10^{8}$ ohm-cm respectively. Voss et al (1983), also reported low resistivity in case of Fe doped PMN. The rapid fall in resistivity in Fe containing samples, for increasing $\mathrm{Fe}$ content and sintering temperature, is due to the increased oxygen vacancies leading to ionic conduction. Similar effect has also been observed by Yokosuka (1995).

\section{Conclusions}

All the compositions selected in the present study form solid solution with PMN. ZnO addition in PMN as well as in PMN-PT improves sinterability. A fairly high relative permittivity maximum obtained for PMZN1 with $T_{\mathrm{c}}=27 \cdot 5^{\circ} \mathrm{C}$ and PMZNT1 with $T_{\mathrm{c}}=34.5^{\circ} \mathrm{C}$ reflected low firing capabilities of these relaxors satisfying the present demand for practical utilization. In contrast, Fe associated solid solutions exhibit high permittivity accompanied with high losses which limit their practical utility.

\section{References}

Bokov V A and Mylnikova I E 1961 Sov. Phys. Solid State 22428

Bouquin O and Lejeune M $1991 \mathrm{~J}$. Am. Ceram. Soc. 741152

Desgardin G, Bali H and Raveau B 1983 Mater. Chem. \& Phys. 8469

Goldschmidt V M 1926 Shrifter Norske Videnskaps-Akad. Oslo: Matemot. Naturuid. Klasse, No 2

Ichionse K and Kato N 1994 J. Appl. Phys. 335423

Landin S M and Schulze W A 1990 J. Am. Ceram. Soc. 73913

Lejeune M and Boilot J P 1985 Mater. Res. Bull. 20493

Ravindranathan P, Komarneni S, Bhalla A and Roy R 1991 J. Am. Ceram. Soc. 742996

Shrout T R and Halliyal A 1987 Am. Ceram. Soc. Bull. 66704

Singh K and Band S A 1996 Ferroelectrics 175193

Smolenskii G A and Agranovskaya A I 1959 Sov. Phys. Solid State 11429

Swartz S L and Shrout T R 1982 Mater. Res. Bull. 171245

Swartz S L, Shrout T R, Schulze W A and Cross L E 1984 J. Am. Ceram. Soc. 167311

Voss D J, Swartz S L and Shrout T R 1983 Ferroelectrics 50203

Wang H C and Schulze W A 1990 J. Am. Ceram. Soc. 73825

Yan M F, Ling H C and Rhodes W 1989 J. Mater. Res. 4930

Yokosuka M 1995 Jpn J. Appl. Phys. 345338 